\title{
FRACTAL DIMENSIONS OF INTERSTELLAR MEDIUM: II. THE MOLECULAR CLOUDS ASSOCIATED WITH THE H II REGION SH 156
}

\author{
Youngung Lee, Miju Kang, Bong Kyu Kim, Jae Hoon Jung, \\ Hyun Goo Kim, Insung Yim, Hyung Woo Kang, and Jihoon Choi \\ Korea Astronomy and Space Science Institute, \\ Hwaam-dong 61-1, Yusong-gu, Taejon, 305-348, Korea \\ E-mail:yulee@kasi.re.kr \\ (Received: December 8, 2008; Accepted: December 24, 2008)
}

\begin{abstract}
We have estimated the fractal dimension of the molecular clouds associated with the $\mathrm{H}$ II region Sh 156 in the Outer Galaxy. We selected the ${ }^{12} \mathrm{CO}$ cube data from the FCRAO CO Survey of the Outer Galaxy. Using a developed code within IRAF, we identified slice-clouds (2-dimensional clouds in velocity-channel maps) with two threshold temperatures to estimate the fractal dimension. With the threshold temperatures of $1.8 \mathrm{~K}$, and $3 \mathrm{~K}$, we identified 317 slice-clouds and 217 slice-clouds, respectively. There seems to be a turn-over location in fractional dimension slope around NP (area; number of pixel $)=40$. The fractal dimensions was estimated to be $D=1.5 \sim 1.53$ for $N P \geq 40$, where $P \propto A^{D / 2}$ ( $P$ is perimeter and $A$ is area), which is slightly larger than other results. The sampling rate (spatial resolution) of observed data must be an important parameter when estimating fractal dimension. Fractal dimension is apparently invariant when varying the threshold temperatures applied to slice-clouds identification.
\end{abstract}

Key words : ISM: cloud - ISM: structure - ISM: fractal dimension - ISM: H II region

\section{INTRODUCTION}

Integrated intensity maps or velocity channel maps of interstellar medium, such as molecular clouds and atomic clouds, have shown irregular hierachical structures, which seem to be similar to each other over a wide range of scales (Scalo 1990; Falgarone et al. 1991). This self-similarity has been interpreted as a signature of underlying fractal geometry, which turbulence plays a major role in the dynamics of interstellar medium. Thus, one way of describing the structure of the molecular and atomic clouds is based on their fractal dimensions: This is a simple topological approach, which consists of characterizing the ISM structure through its fractal dimension. Fractal dimension $D$ of a molecular cloud boundary can be determined from the perimeter $(P)$-area $(A)$ relation of a map; $P \propto A^{D / 2}$. Many observational studies of the interstellar medium have shown that the boundaries of clouds have projected dimensions of a range of $D=1.2 \sim 1.5$ (Sanchez et al. 2007). The range of the fractal dimensions seems to be also valid for IRAS cirrus clouds (Bazell \& Desert 1988), molecular clouds (Lee 2004; Dickman et al. 1990), high-velocity clouds (Vogelaar \& Walker 1994) and so forth. Falgarone, Phillips \& Walker (1991) observed several molecular clouds at two different distances at high angular resolution using several transitions of CO molecule. Their final estimate of the fractal dimension was $D=1.36$. All the fractal dimension studies are for the clouds in the Inner Solar circle, and no studies had been reported on the clouds located in the outside solar circle, especially molecular associated with $\mathrm{H}$ II region, where the environment could be quite different from that of the Inner Galaxy region.

Recently, Lee (2004) reported that the fractal dimension of the molecular clouds in the antigalactic center is almost the same as those of the Inner Galaxy clouds, though he claimed that the results may depend on the spatial resolution of the observed data.

In this paper, we will examine the practical definition of fractal dimension more closely, using a wellsampled database of the molecular clouds in the Outer Galaxy. We will use the molecular cloud complex associated with an $\mathrm{H}$ II region Sh156. In section II, we will describe the database, and we will define the fractal dimension in section III. In section IV, we will discuss the results. We summarize our results in the final section.

\section{DATA SELECTION}

We obtained ${ }^{12} \mathrm{CO}(J=1-0)$ data for study of fractal dimension estimation from the FCRAO CO Survey of the Outer Galaxy (OGS; Heyer et al. 1998). OGS is comprised of $1,696,800$ spectra sampled every $50^{\prime \prime}$ covering $l=102 .^{\circ} 49$ to $141 .{ }^{\circ} 54$, and $b=-3 .^{\circ} 03$ to $5 .^{\circ} 41$. The $V_{\text {LSR }}$ ranges from -153 to $+40 \mathrm{~km} \mathrm{~s}^{-1}$ sampled every $0.81 \mathrm{~km} \mathrm{~s}^{-1}$ with a velocity resolution of 0.98 $\mathrm{km} \mathrm{s}^{-1}$. This survey provides the highest spatial dy- 


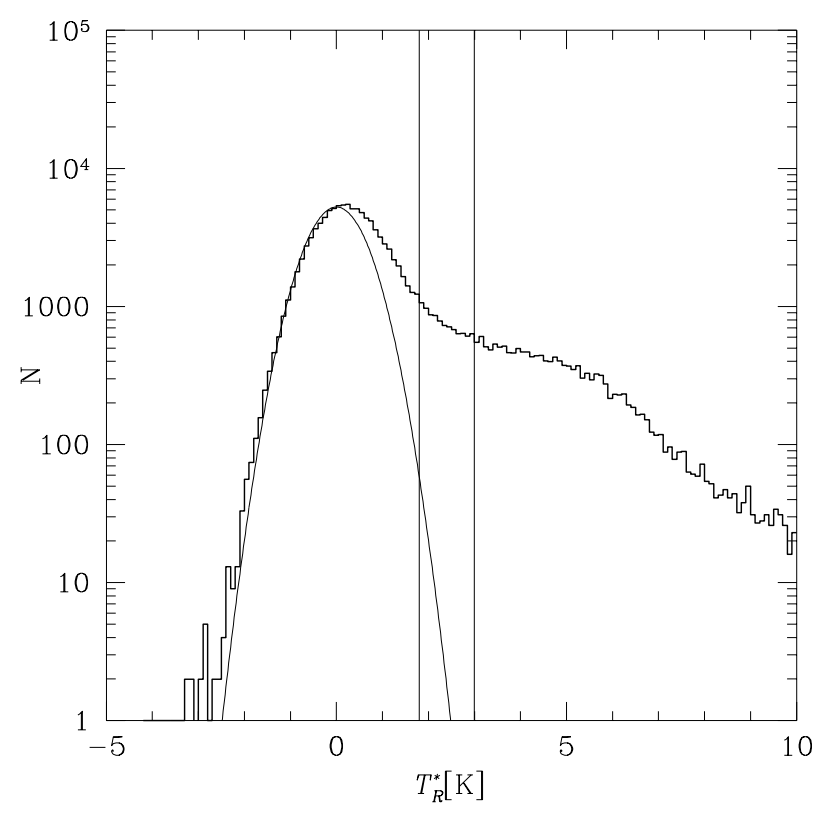

Fig. 1.- Distribution of brightness temperatures in all channels of the spectra of the whole region selected is represented on a logarithmic scale. The shape of the negative brightness temperature shows a almost complete Gaussian distribution, which gives an rms noise level. A fitted Gaussian curve is represented with an estimated $\sigma=0.6 \mathrm{~K}$. Two straight lines are the $3 \sigma$ and $5 \sigma$, which are used as threshold temperatures.

namic range imaging of molecular interstellar medium ever obtained except the recent ${ }^{13} \mathrm{CO}$ Galactic Ring Survey (Jackson et al. 2006). Thus, the ${ }^{12} \mathrm{CO}$ Outer Galaxy Survey database is also ideally suited for our study of fractal dimension in terms of spatial and velocity resolution. The previous CO surveys have had either coarse angular resolution or have been restricted to the first quadrant of the Galaxy, where molecular clouds are blended in space and velocity, which has been making it difficult to identify and to extract the complicated structure of the molecular clouds in the Inner Galaxy.

The selected region is about $68^{\prime} \times 68^{\prime}$ area centered on the $\mathrm{H}$ II region Sh $156(l, b)=\left(110^{\circ} .0,0^{\circ} .1\right)$, which is $80 \times 80$ in pixel units in $(l, b)$ space. We use only 19 channels in the line of sight, which corresponds to the velocity range of the associated clouds, -57.7 to -43.1 $\mathrm{km} \mathrm{s}^{-1}$. Sh 156 is a compact H II region located in Perseus spiral arm. The physical properties of associated molecular gas has been recently studied by Kang $\&$ Lee (2005). This selected region in $(l, b, v)$ space is slightly larger than that of previous study on Sh 156 (Kang \& Lee 2005).

This object has been known to be very intriguing for the infrared and ultraviolet astronomers as noted by Kang \& Lee (2005); Heydari-Malayeri et al. (1980) reported that the observed ionization structure seems to be in good agreement with the $\mathrm{O} 7 \mathrm{~V}$ spectral type of the exciting star in their optical wavelengths study and that the ionizing star lies outside the molecular cloud. We selected this region as our target object as it is well isolated from other molecular gas in this direction, and associated with a compact $\mathrm{H}$ II region, which can seriously affect the morphology of the surrounding molecular gas, and the pixels numbers of the observed region is large enough. The integrated intensity map and the velocity channel maps were shown in detail by Kang \& Lee(2005). The rms noise level of the selected region was found to be $0.6 \mathrm{~K}$ per channel of $0.81 \mathrm{~km} \mathrm{~s}^{-1}$. A histogram of the number of $l, b, v$ data points (voxels) versus brightness temperature of the ${ }^{12} \mathrm{CO}$ data for the whole voxels of the target region is shown in Figure 1 in logarithmic scale. The negative part of the Figure 1 shows a well-defined Gaussian distribution, which shows the consistency of noise level of the region selected. A fitted Gaussian curve is represented; small number of voxels at the skirt of the curve are just spurious noise which can be ignored. Two straight lines are the $3 \sigma$ and $5 \sigma$, which will be used as threshold temperatures (see below).

\section{ESTIMATE OF FRACTAL DIMENSION}

The measurement of the fractal dimension of the molecular clouds is one simple way to characterize the fractal structure. The best way to calculate the fractal dimension of an object would be possible when we know the three dimensional structure. For the interstellar medium, however, it is impossible to get threedimensional structure. For example, in $(l, b, z)$-space where $z$ is the direction of line of sight, which can't be observed directly at present. All images obtained are projected on two-dimensional space, whether it is integrated intensity map or channel maps. Several factors may contribute to affect on estimating their fractal dimensions, when projected integrated intensity maps are used; spatial resolution and data sensitivity (rms noise level) would be the most influential (Lee 2004). Moreover, the number of pixels becomes an issue when threshold levels gets higher when using integrated intensity map. With higher threshold values, the number of pixels would be much smaller that of initial lower threshold values, which may affect the slope of the perimeter-area relationship (see below). Thus, instead of using the integrated intensity map, we generated slice-clouds, similar way to get channel maps, and estimated the area and perimeter of each slice-cloud. We identify them in two dimensional space; we choose to slice into velocity direction from a cube data set in $(v, l, b)$ space. The "slice-clouds" are the part of clouds sliced through each channel.

However, all the data points of the sliced-clouds or integrated intensity maps are comprised of the units of pixels, which can be transformed to $\mathrm{pc}$ and $\mathrm{pc}^{2}$. Thus, when estimating perimeter and area of a cloud, we should just count the pixel numbers; all the pixels 


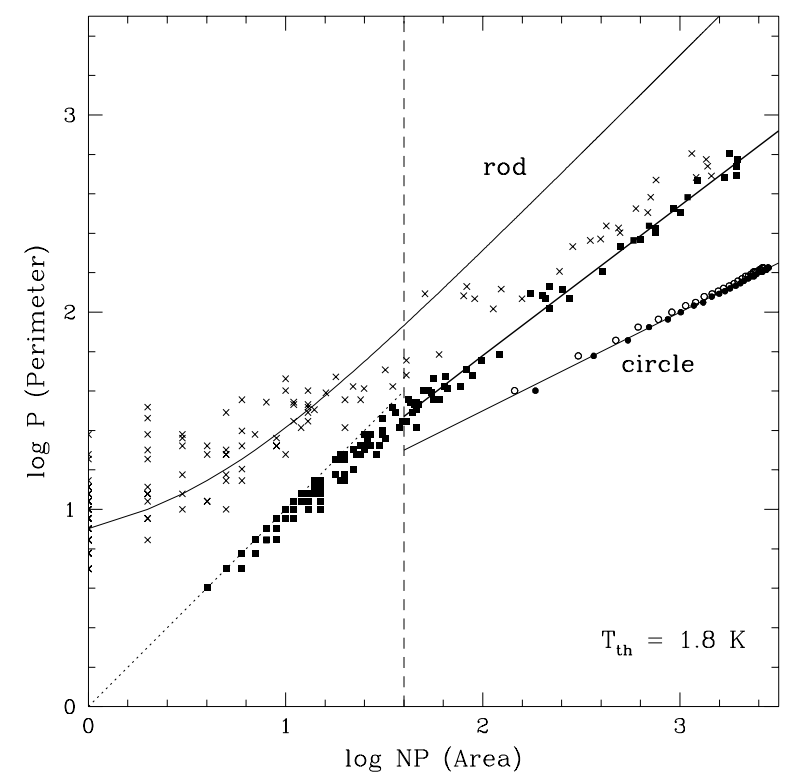

Fig. 2.- Log-log plot of measured perimeter versus area in units of pixels in $(l, b)$ space for the slice-clouds using a threshold temperature of $1.8 \mathrm{~K}$. We present the filled squares when including the boundary pixels to the areas, and $\mathrm{x}$ marks when excluding the boundary pixels, for the target clouds. Two solid lines are indicating the arbitrary elongated rod-type clouds and circular clouds for comparison. The filled circles on the circle line are for the case when including the boundary pixels to the areas, and the open circles for the case when excluding the boundary pixels, for circular-type clouds. Dashed line represents the pixel number at $N P=40$, and a bisector least-square fit (a solid line) for filled squares $(N P=40)$ is represented. The dotted line marks an upper limit for the clouds with smaller pixel numbers $N P<40$, and its slope is $1(D=2)$.

at the boundary of the cloud as perimeter, and all the pixels inside the boundary.

As mentioned in the previous paper (Lee 2004), we developed and revised a code, which is working as a user task within IRAF, to effectively identify the clouds at each velocity slice (or each channel) from the 3dimensional cube data set. Using the ${ }^{12} \mathrm{CO}$ cube data we define a cloud to be an object composed of all pixels in longitude, and latitude that are simply connected and that lie above some threshold temperature. Ideally, one would like to define clouds with a $0 \mathrm{~K}$ threshold temperature. However, $0 \mathrm{~K}$ threshold temperature is impractical in view of the noise level in the spectra. On the other hand, with too high a threshold temperature, regions are severely truncated, and it is impossible to obtain a reliable estimate of the size. To define clouds we should choose a reasonable threshold temperature. Above the arbitrary threshold temperature, only those clouds with 4 or more pixels $(l, b)$ space were retained.

There could be two different definitions of area as

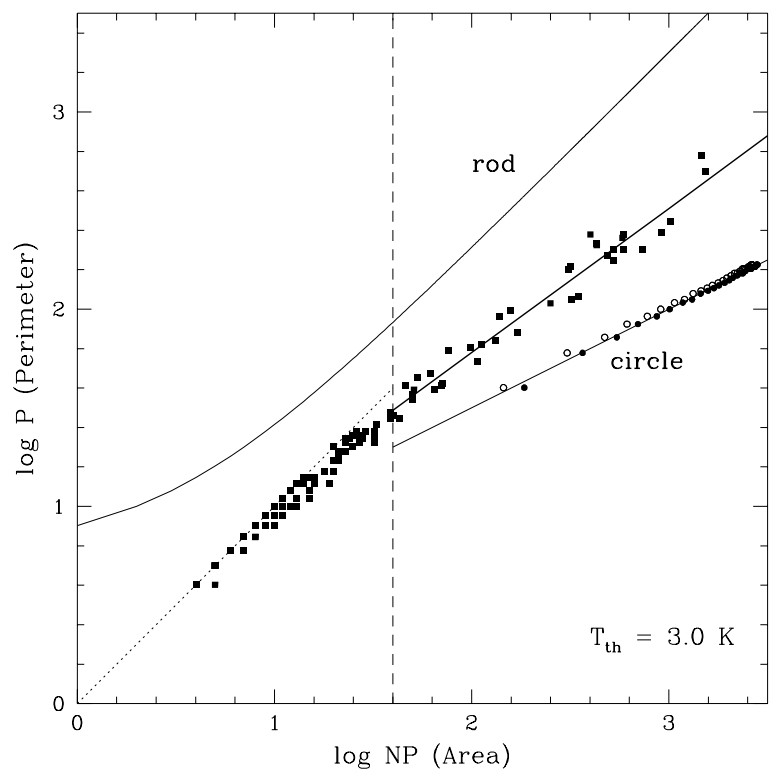

Fig. 3.- Log-log plot of measured perimeter versus area in units of pixels in $(l, b)$ space for the slice-clouds using a threshold temperature of $3 \mathrm{~K}$. Other symbols are the same as Figure 2. The $\mathrm{x}$ marks are not plotted in this figure, as they show the same trend as in Figure 2.

mentioned in Lee (2004). The first definition of area is simply integration of all the unit pixels, or alternatively, number of pixels above a threshold temperature. The second one is excluding the pixels on the boundary, which is perimeter. In the second case, the perimeterarea relation could be biased, as their areas would be estimated much smaller especially for the small-pixel clouds. This may be a simple comment, but can often be ignored when estimating the fractal dimension. Maybe this might be the reason for wider range of fraction dimension of $1.2 \sim 1.5$ (Sanchez et al. 2007). We represented it in Figure 2, showing how erroneously they are dispersed and thus, that its estimate of fractal dimension can be different.

\section{RESULTS and DISCUSSION}

We used two threshold temperatures of $3 \sigma$ (1.8 $\mathrm{K})$ and $5 \sigma(3.0 \mathrm{~K})$, and identified 317 and 217 sliceclouds for the target region. Two threshold temperatures (solid lines) are shown in Figure 1. The emission sum above $3 \sigma$ of the rms noise level is found to be $82.0 \%$ of the total emission of the selected region, and $65.1 \%$ above $5 \sigma$. Figures 2 and 3 show a relationship between the perimeters and the areas of the slice-clouds in logarithmic scale. The perimeters $(P)$ and areas $(N P)$ are in units of pixels. Two solid lines are indicating the arbitrary elongated rods and circles (slice of a spherical clouds). The extreme cases for any cloud's structure could be highly elongated (rod), or 
perfectly circular (ellipsoid or spheroid). Their fractal dimension is $D=2$ for rods (straight part), and $D=1$ for circles. The filled circles presented on the circle are the case if we include pixels at the boundary when estimating areas. The open circles are for the case when excluding pixels at the boundary. Circular clouds are supposed to be lining on a straight line with a fractal dimension of $D=1$, when including the boundary pixels into areas. However, if the number of pixels are small enough, its position is slightly off the straight line. Likewise, slice-clouds with a small number of pixels tends to be similar to rods lining up with a upper limit of fractal dimension of $D=2$, which represented as dotted lines up to $N P=40($ or $\log N P=1.6)$ in Figures 2 and 3.

In the meanwhile, there seems to be a turn-over location in slope in Figure 2 around $N P=40$, which is expected as the smaller number of pixels involved may give a different result. Thus, we estimated clouds' fractal dimensions for $N P \geq 40$. Only 47 slice-clouds are larger than 40 in pixel number for $T_{t h}=1.8 \mathrm{~K}$ (Case A), and 38 slice-clouds for $T_{t h}=3.0 \mathrm{~K}$ (Case B). The number of pixels (areas) of the largest slice clouds are 1,955 for Case A, and 1,529 for Case B.

Figures 2 and 3 show a good correlationship between two parameters for both cases. A bisector fit (Isobe et al. 1990; Lee, Snell \& Dickman 1990) applied to each case yields a best fit line.

For Case A:

$$
\log (\mathrm{P})=1.532( \pm 0.048) / 2 \log (\mathrm{NP})+0.243( \pm 0.016)
$$

and the Case B:

$$
\log (\mathrm{P})=1.496( \pm 0.050) / 2 \log (\mathrm{NP})+0.279( \pm 0.008)
$$

The slopes for two cases are found to be almost the same; $D=1.5 \sim 1.53$. We noticed that the intercepts are not important, as they are related to the resolution (pixel size) of the observed data. The estimated areas and and perimeters are based on the pixel units, rather than the actual size in arcminute units. Even though we corroborate this (using the actual perimeters and areas in arcminute and square arcminute units), the slope would not be changed. Only the intercepts will be changed. Even though we change the threshold values, such as $7 \sigma$, or $10 \sigma$, there is no big change in estimated fractal dimensions.

Recently, Sanchez et al. (2007) pointed out that a projection $\left(D_{\text {pro }}\right)$, which is the same symbol as $D$ in this paper, is a totally different operation and the result $D_{\text {pro }}=D_{f}-1$ does not have to be expected for the projected fractal dimension, where $D_{f}$ three-dimensional fractal dimension. This comment is concerned with Mandelbrot (1983)'s theory that the intersection of a fractal with dimension $D_{f}$ and a plane gives another fractal with dimension given by $D_{f}-1$. They, thus, interpreted that the projection of a cloud with $D_{f} \sim 2.35$ would give rise to a compact shadow that may not be a fractal, but emphasized that the relationship between the fractal dimension of this projection and that of its boundary is not clearly established. In addition, Sanchez et al. claimed that $D_{f}=2.6 \sim 2.7$ and that it is matching with their simulated fractional dimension $D_{\text {pro }}=1.35$. Stutzki et al. (1998) have, however, argued that this numerical agreement could be coincidental; in fact, they used fractional Brownian motion structures to analyze the observed ISM properties, concluding that the index of power spectrum that the best reproduces the observed characteristics is $\sim 2.8$, which is three-dimensional fractal dimension. This is quite different from above-mentioned Mandelbrot's theory.

There are many kinds of molecular clouds in the interstellar medium, such as quiescent, violent or star forming clouds, shocked gas, and cometary clouds etc. Williams et al. (2000) argued that the invariance of the fractal dimension of these clouds may imply selfsimilar structure of the molecular clouds, and turbulent motion within them; A self-similar object is exactly or approximately similar to a part of itself. If the target clouds are fractal, they are called to be self-similar. Most observed molecular clouds has the highly supersonic linewidths, and this probably implies turbulence motion in them, for which one would naturally expect a fractal structure (Mandelbrot 1983). In addition, invariance of fractal dimension $D$ from cloud to cloud might be related to the similarity in mass spectrum index (Kramer et al. 1998). However, considering Mandelbrot (1983)'s theory of $D_{\text {pro }}=D_{f}-1$ and Sanchez et al. (2007)'s three-dimensional fractal dimension of 2.6 for Orion A, our result $(D=1.5)$ is closer than other results of 1.35 . The relationship between $D_{f}$, $D_{\text {pro }}$, and the physical processes determining the interstellar medium structure is still an open issue. Our target region is molecular clouds associated with an $\mathrm{H}$ II region in the Outer Galaxy. The obtained fractal dimension is slightly larger than the range $(1.2 \sim 1.5$ of many other studies, or in its upper limit. As this is just one case of higher dimension, more study is required for more clouds in the Outer Galaxy with a wellsampled database. Or alternatively, finer sampling rate and more sensitive observations may matter, which we will investigate in further study.

\section{SUMMARY}

We have estimated the fractal dimension of the molecular clouds associated with the H II region Sh 156 in the Outer Galaxy. We selected the ${ }^{12} \mathrm{CO}$ cube $(v, l, b)$ database from the FCRAO CO Survey of the Outer Galaxy. Using a developed code within IRAF, we identified slice-clouds with two threshold temperatures, instead of using integrated intensity maps of the clouds, to estimate the fractal dimension. With the threshold temperatures of $1.8 \mathrm{~K}$, and $3 \mathrm{~K}$, we identified 317 sliceclouds and 217 slice-clouds, respectively. There seems to be a turn-over location in fractional dimension slope around NP (area) $=40$. Thus, we estimated clouds' 
fractal dimensions for $N P \geq 40$. Only 47 slice-clouds are larger than 40 in pixel number for $T_{t h}=1.8 \mathrm{~K}$ (Case A), and 38 slice-clouds for $T_{t h}=3.0 \mathrm{~K}$ (Case B). The number of pixels (areas) of the largest slice clouds are 1,955 for Case A, and 1,529 for Case B. We included the number of perimeter into area, which may induce more reliable estimate of fractal dimension of the clouds than excluding it from the area estimate. The fractal dimensions was estimated to be $D=1.5 \sim 1.53$, where $P \propto A^{D / 2}$, which is slightly larger than other results, or in the upper limits of the range of fractal dimension of other studies. The sampling rate (spatial resolution) of observed data must be an important parameter when estimating fractal dimension; As can be seen in Figures 2 and 3, the smaller number of pixels(area) may cause a quite different estimate of fractal dimension. Enough number of data points is required for more reasonable estimate of fractal dimension of the clouds. Fractal dimension is apparently invariant when varying the threshold temperatures applied to cloud identification.

\section{ACKNOWLEDGEMENTS}

This work was supported by grant R01-2003-00010513-0 from the Basic Research Program of the Korea Science and Engineering Foundation (KOSEF).

\section{REFERENCES}

Bazell, D. \& Desert, F. X., 1988, Fractal structure of interstellar cirrus, ApJ, 333, 358

Blitz, L., Lockman, E. J., \& Tschope, R., 1990, Outer Galaxy. Lecture Notes in Physics No. 306, SpringerVerlag, Berlin.

Dickman, R. L., Horvath, M. A., Margulis, M., 1990, A Search for Scale-Dependent Morphology in Five Molecular Cloud Complexes, ApJ, 365, 586

Falgarone, E., Phillips, T. G., \& Walker, C. K., 1991, The Edges of Molecular Clouds - Fractal Boundaries and Density Structure, ApJ, 378, 186

Heydari-Malayeri, M., Testor, G., \& Lortet, M. C., 1980 , Interaction of hot stars and the interstellar matter. $\mathrm{X}$ - Morphology, excitation, and structure of the bright galactic nebula Sh2-156/IC 1470/, A\&A, 84, 154

Heyer, M. H., Brunt, C., Snell, R. L., Howe, J. E., Schloerb, F. P., \& Carpenter, J. M., 1998, The Five College Radio Astronomy Observatory CO Survey of the Outer Galaxy, ApJS, 115, 241

Isobe, T., Feigelson, E. D., Akritas, M. G., \& Babu, G. J., 1990, Linear Regression in Astronomy, ApJ, 364, 104

Kang, M. \& Lee, Y., 2005, A Study of Molecular Cloud Associated with the H II Region Sh 156, JKAS, 38, 33

Kramer, C., Stutzki, J., Rohrig, R., Corneliussen, U., 1998, Clump Mass Spectra of Molecular Clouds, A\&A, 329, 249

Lee, Y., Snell, R. L., \& Dickman, R. L., 1990, Analysis of ${ }^{12} \mathrm{CO}$ and ${ }^{13} \mathrm{CO}$ Emission in a Three Square Degree
Region of the Galactic Plane between $l=23^{\circ}$ and $25^{\circ}$, ApJ, 355, 536

Lee, Y., 2004, Fractal Dimensions of Interstellar Medium: I. The Molecular Clouds in the Antigalactic Center, JKAS, 32,1

Mandelbrot, B. B., 1983, The Fractal Geometry of Nature, San Francisco: Freeman.

Sanchez, N., Alfaro, E. J., \& Perez, E., 2005, The Fractal Dimension of Projected Clouds, ApJ, 625, 849

Scalo, J., 1990, Perception of interstellar structure - Facing complexity in Physical processes in fragmentation and star formation, ed. R. Capuzzo-Dolcetta, C. Chiosi, \& A, Di Fazio (Dordrecht: Kluwer), ASSL, 162, 151,

Scoville, N. Z., Yun, M. S., Clemens, D. P., Sanders, D. B., and Waller, \& W. H., 1987, Molecular Clouds and Cloud Cores in the Inner Galaxy, ApJS, 63, 821

Stutzki, J., Bensch, F., Heithausen, A., Ossenkopf, V., \& Zielinsky, M., 1998, On the Fractal Structure of Molecular Clouds, A\&A, 226, 697

Vogelaar, M. G. R. \& Wakker, B. P. 1994, Measuring the fractal structure of interstellar clouds, A\&A, 291, 557

Williams, J. P., Blitz, L.,\& McKee, C. F., 2000, Protostars and Planets IV (Book - Tucson: University of Arizona Press; eds Mannings, V., Boss, A.P., Russell, S. S.), p. 97. The Structure and Evolution of Molecular Clouds: from Clumps to Cores to the IMF. 